\title{
Mother and Adolescent Reports of Associations Between Child Behavior Problems and Mother-Child Relationship Qualities: Separating Shared Variance from Individual Variance
}

\author{
William J. Burk • Brett Laursen
}

Published online: 9 March 2010

(C) The Author(s) 2010. This article is published with open access at Springerlink.com

\begin{abstract}
This study contrasts results from different correlational methods for examining links between mother and child ( $N=72$ dyads) reports of early adolescent $(M=$ 11.5 years) behavior problems and relationship negativity and support. Simple (Pearson) correlations revealed a consistent pattern of statistically significant associations, regardless of whether scores came from the same reporter or from different reporters. When correlations between behavior problems and relationship quality differed, withinreporter correlations were always greater in magnitude than between-reporter correlations. Dyadic (common fate) analyses designed for interdependent data decomposed withinreporter correlations into variance shared across reporters (dyadic correlations) and variance unique to specific reporters (individual correlations). Dyadic correlations were responsible for most associations between adolescent behavior problems and relationship negativity; after parti-
\end{abstract}

Support for this research was provided to Brett Laursen by the US National Institute of Child Health and Human Development (HD33006) and the US National Science Foundation (0923745). We are grateful for the cooperation of the administration, staff, teachers, parents, and students in the Broward and Miami-Dade Public School Systems.

W. J. Burk

Behavioural Science Institute, Radboud University Nijmegen, Nijmegen, The Netherlands

\section{B. Laursen}

Department of Psychology, Florida Atlantic University,

Boca Raton, FL, USA

e-mail: laursen@fau.edu

\section{W. J. Burk ( $\square)$}

Developmental Psychology, Behavioural Science Institute, Radboud University Nijmegen,

PO Box 9104, 6500 HE Nijmegen, The Netherlands

e-mail: w.burk@psych.ru.nl tioning variance shared across reporters, no individual correlations emerged as statistically significant. In contrast, adolescent behavior problems were linked to relationship support via both shared variance and variance unique to maternal perceptions. Dyadic analyses provide a parsimonious alternative to multiple contrasts in instances when identical measures have been collected from multiple reporters. Findings from these analyses indicate that samereporter variance bias should not be assumed in the absence of dyadic statistical analyses.

Keywords Adolescent behavior problems .

Mother-child relationships $\cdot$ Nonindependent data .

Common fate model

Adjustment difficulties pose a special challenge to family relationships. Poor quality relationships with parents, especially mothers, are common among maladjusted adolescents. Links between adolescent behavior problems and parent-child relationships have been documented (Collins and Steinberg 2006), but little is known about how specific syndromes map onto specific relationship attributes. Complicating matters is that fact that mothers and adolescents have correlated yet distinct perceptions of their relationship (Laursen and Collins 2009). The correlated nature of mother and child reports makes it difficult to disentangle associations shared by mother and child from associations that are unique to each. Shared reporter variance is of particular concern because it may overstate links between variables. In the present study, we contrast results from conventional correlation approaches with those from procedures designed for use with nonindependent data. The latter describes associations between adolescent behavior problems and mother-child relationship quality with components that partition variance 
unique to mothers' perceptions and variance unique to adolescents' perceptions from variance shared by mothers' perceptions and adolescents' perceptions.

In the classic Isle of Wight study, early adolescents diagnosed with a psychiatric disorder described levels of withdrawal, communication difficulties, and altercations with parents that were two to four times higher than those reported by nondisordered youth (Rutter et al. 1976). More recent comparisons confirm that families of adolescents with behavior problems experience more frequent disagreements (Forehand et al. 1987) and perceive parent-child relationships to be less supportive (Tremblay et al. 2004) than families of adolescents without behavior problems. Community samples confirm associations between adolescent adjustment and the quality of parent-child relationships (Caples and Barrera 2006). Thus, adolescent behavior problems have been tied to poor family relationships among clinical and nonclinical samples alike. Most studies are limited to global measures that describe behavior problems in terms of internalizing and externalizing behaviors. When specific syndromes are considered, there is evidence that adolescent perceptions of maternal warmth and support are tied to depression and anxiety, and that adolescent perceptions of maternal hostility and negativity are linked to aggression and conduct problems (Adams and Laursen 2007; Ge et al. 1996). These studies extend the widely held view that child behavior problems are linked to relationships with parents and suggest that different aspects of relationships may be tied to different indices of adjustment.

Methodological obstacles complicate conclusions. Mothers and adolescent children hold distinct yet overlapping views of one another and of their relationship. Moderate levels of agreement characterize reports concerning social interactions and relationship perceptions (Noller and Callan 1988; Tien et al. 1994), as well as adolescent behavior problems (Achenbach et al. 1987; De Los Reyes and Kazdin 2005). Correlations between reporters are not high (e.g., $r=0.25$ between mother and child reports of adolescent behavior problems; Achenbach et al. 1987), but they are of a sufficient magnitude that variables ought not be considered independent. This raises a challenging analytic problem, because standard parametric statistics (e.g., ANOVAs and linear regressions) cannot easily accommodate nonindependent data; most are predicated on the assumption that individual observations are uncorrelated. Violations of the independence assumption bias standard errors of estimates, inflating the significance of standardized coefficients (Kenny 1995). To avoid this problem, predictor variables may be limited to reports from only one member of the dyad, but this solution is less than satisfactory for a number of reasons. First, it divides perceptions that are shared by partners into perceptions that appear to be unique to each partner. The result is an undue emphasis on dyadic differences at the expense of dyadic similarity (Laursen 2005). Second, analyzing data separately by reporters gives rise to considerable variation in the pattern and the magnitude of associations. Assertions that adolescent behavior problems are correlated with parentchild relationship quality are generally followed by qualifications concerning reporters. For instance, the degree to which adolescent externalizing symptoms are associated with maternal support vary according to the reporter (Barrera et al. 1993; Tesser et al. 1989). The most robust links are found when either the adolescent or the mother is the source of reports on both the independent and the dependent variable. The conventional interpretation of this finding holds that same-reporter biases inflate associations. No studies have examined the possibility that the variance described in single-reporter accounts may actually be shared by both reporters.

The correlated nature of close relationship partners' perceptions requires analytic techniques capable of accounting for various interdependencies inherent in dyadic data (Laursen et al. 2008; Kenny et al. 2006). Instances in which both relationship partners report on the same variable, such as mother and child reports of adolescent behavior problems, require methods that are capable of disentangling variance shared across reporters from variance unique to each reporter. The common fate model (Gollob 1991; Gonzalez and Griffin 1999; Kenny and La Voie 1985) is particularly well suited to this task, because it partitions dyadic (or group) associations into shared and unique components. Figure 1 presents a conceptual model of the shared and unique associations between mother and child reports of adolescent behavior problems and mother-child relationship quality. The dyadic correlation describes the linear association between shared perceptions (i.e., correlation between the latent variable representing child and maternal reports of adolescent behavior problems and the latent variable representing child and maternal reports of relationship quality). Individual correlations describe the association that is unique to mothers (i.e., correlation between maternal reports of the child's behavior problems and maternal reports of the mother-child relationship) and the association that is unique to children (i.e., correlations between child reports of their own behavior problems and child reports of the mother-child relationship). These three associations may be interpreted as partial correlations because all are estimated in a single analysis.

In the present study, we examine associations between mother and adolescent reports of child behavior problems (aggressive behaviors, anxiety/depression, attention problems, delinquent behaviors, social problems, somatic complaints, thought problems, and withdrawn behaviors) and mother-child relationship quality (perceived negativity and support). We contrast results from conventional analytic approaches, represented by simple within- and 
between-reporter correlations, with results from the common fate model, represented by dyadic and individual partial correlations. We expected simple within-reporter correlations to be greater in magnitude than simple between-reporter correlations, which might ordinarily be interpreted as evidence that same-reporter variance inflated associations between adolescent behavior problems and mother-child relationships quality. We predicted that common fate analyses would reveal these associations to be less a function of same-reporter bias than of common variance arising from perceptions shared by mothers and children. Put simply, we expected individual correlations to be smaller in magnitude than dyadic correlations.

\section{Method}

\section{Participants}

The participants were 72 young adolescents (42 females and 30 males) and their biological mothers. Adolescents ranged in age from 11 to 13 years old $(M=11.5)$. Participants included 13 non-Hispanic African American dyads, 40 non-Hispanic Anglo American dyads, and 19 Hispanic American dyads. There were 59 dyads from two biological parent families, 7 dyads from biological mother and step-father families, and 6 dyads from single mother families. Socioeconomic status (SES) was assessed with the Hollingshead (1975) four-factor index, derived from mother reports of parent education and occupation. Out of a potential range of 8 to 66 , SES scores in the present study ranged from 11 to $64(M=40.6, S D=10.3)$.

\section{Instruments}

Adolescents were administered questionnaires in small group sessions in school. Research assistants read the written instructions for each survey aloud. Mothers were mailed questionnaires (in English and Spanish), a letter describing each, and a postage paid return envelope. All variables used in the dyadic analyses were centered by subtracting the score of each member of the dyad from the mean of the dyad (Cook and Kenny 2005).

Adolescent Behavior Problems Adolescents completed the Youth Self-report (YSR) and mothers completed the Child Behavior Checklist (CBCL) (Achenbach 1991). These instruments assess adolescent behaviors during the previous six months in 8 different domains: (a) aggressive behaviors, (b) anxiety/depression, (c) attention problems, (d) delinquent behaviors, (e) social problems, (f) somatic complaints, (g) thought problems, and (h) withdrawn behaviors. Subscales included 7 to 20 items, rated on a scale ranging from 0 (never) to 2 (often). The internal reliability of these subscales was adequate (mother $\alpha=0.64$ to 0.79 , adolescent $\alpha=0.70$ to $0.87)$. Subscales were combined to form three general indices of behavior problems. Externalizing symptoms include aggressive behaviors and delinquent behaviors. Internalizing symptoms include anxiety/depression, somatic complaints, and withdrawn behaviors. Total symptoms include all eight subscales. The internal reliability of these combined scales was good (mother $\alpha=0.84$ to 0.88 and adolescent $\alpha=0.84$ to 0.85 ). Individual scores were positively skewed, with standardized values (skewness statistic divided by its standard error) ranging from 4.31 to 8.33 for mothers and from 2.90 to 6.68 for adolescents. A square root transformation proved to be the most effective method of reducing the skew of each distribution (standardized values of transformed scores ranged from -0.99 to 1.66 for mothers and from -1.84 to 1.63 for adolescents). Centered transformed scores were used in structural equation models and correlations; mean item scores were used in $t$-tests. Several studies have demonstrated the distinctiveness and test-retest reliability of these subscales during the adolescent years, and their strong concurrent associations with clinical assessments (e.g., Achenbach et al. 1989; Edelbrock and Costello 1988).

Mother-Child Relationship Quality Adolescents and mothers completed the Network of Relationships Inventory (NRI: Furman and Buhrmester 1985). This instrument contains 33 items rated on a scale ranging from 1 (little or none) to 5 (the most). Factor analyses of ratings of parent-adolescent relationships yield three distinct subscales (Adams and Laursen 2007; Furman 1996): support, negativity, and relative power. Support includes 24 items that describe admiration, affection, companionship, instrumental aid, intimacy, nurturance, reliable alliance, and satisfaction (e.g., How satisfied are you with your relationship with this person?). Negativity includes 6 items that describe annoying behaviors and conflict (e.g., How much do you and this person get on each other's nerves?). The third factor, a 3 item subscale describing relative power, was not included in the present investigation. Internal reliability was adequate for the support and negativity subscales (mother $\alpha=0.77$ and 0.81 , adolescent $\alpha=0.87$ to 0.88). Item scores were averaged for each factor. Relationship support scores were negatively skewed. A cubed transformation proved to be the most effective method of reducing the skew of the support distributions (standardized values of transformed scores were -1.84 for mothers and -2.02 for adolescents). Centered transformed scores of support were used in structural equation models and correlations; centered raw scores of negativity were used in all analyses. Mean item scores were used for both measures in $t$-tests. NRI reports of support and negativity are stable across the early adolescent years (Hafen and 
Laursen 2009) and have been validated with observations of parent-child interactions (Furman and Buhrmester 2009; Gavin and Furman 1996).

\section{Procedure}

Adolescents were recruited from 6th grade classes selected by school administrators as representative of the entire school population. In each classroom, teams of research assistants described the study, informing students that their responses would be kept anonymous and confidential, and that no information would be shared with teachers, parents, police, or anyone else. Of these students, assent and parental consent forms were collected from a total of 250 youth. Participation rates ranged from $40 \%$ to $74 \%$ within schools, which is comparable to those found in similar studies of diverse youth (e.g., Silk et al. 2003). Mothers of these youth were also invited to participate in a separate phase of the investigation, and 72 biological mothers completed and returned the surveys. Mothers completed surveys an average of 60 days $(S D=37.4)$ after adolescents. Time between mother and child reports was uncorrelated with all study measures (average $r=$ $0.05, p=0.67)$. A one-way MANOVA was performed with those completing surveys less than 4 weeks apart $(n=18)$, those completing surveys 4 to 12 weeks apart $(n=33)$, and those completing surveys more than 12 weeks apart $(n=$ 21) as the between-subjects factor. Mother and child reports of adolescent behavior problems (CBCL and YSR scales and subscales) and relationships quality (negativity and support) were the dependent variables. There were no statistically significant differences between the groups (Wilk's lambda= $0.40, p=0.34$ ).

A series of chi-square analyses examined whether the 72 adolescents with mother reports and the 178 adolescents whose mothers did not participate differed on sex, ethnicity, and household structure. These analyses revealed that adolescents with mother reports were more apt to be Anglo American than adolescents without mother reports, $\chi^{2}(2)=$ $30.27, p<0.001$. There were no sex or household structure differences. Independent $t$-tests examined differences between adolescents with and without mother reports on SES, adolescent reports of behavior problems (YSR scales and subscales) and mother-child relationship quality (negativity and support). These analyses revealed differences on SES, $t(248)=-2.37, p=0.019$; and withdrawn behaviors, $t$ $(248)=2.33, p=0.021$. Adolescents with mother reports were from higher SES households $(M=40.6, S D=10.3)$ and reported less withdrawn behaviors $(M=0.31, S D=0.24)$ than those without mother reports (SES, $M=37.25, S D=$ 10.51; withdrawn behaviors, $M=0.71, S D=0.49$ ). There were no mean-level differences between groups on any other study variables. Differences between adolescents whose mothers participated and those whose mothers did not participate in correlations between youth self-reports of behavior problems and youth self-reports of mother-child relationship quality did not arise at levels greater than chance ( 2 out of $40, p=0.05$ ) in $r$-to- $z$ correlation contrasts.

\section{Plan of Analysis}

Preliminary analyses describe differences and similarities between mother and child reports. Paired $t$-tests examine dyadic differences, contrasting mother and adolescent reports of adolescent behavior problems and relationship quality. Intraclass correlations establish interdependence (dyadic similarity) between adolescent reports and mother reports, a prerequisite to dyadic data analyses.

To illustrate findings from conventional statistical approaches, we conducted simple (Pearson) correlations between indices of adolescent behavior problems and mother-child relationship support and negativity. Simple correlations were conducted within reporters, using maternal reports of adolescent behavior problems and relationship quality in one set of analyses and child reports of adolescent behavior problems and relationship quality in another set of analyses. Within-reporter correlations are compared using a test for correlated, yet nonoverlapping correlations (Raghunathan et al. 1996). Simple correlations were also conducted between reporters: child-mother correlations included child reports of adolescent behavior problems and maternal reports of relationship quality; mother-child correlations included maternal reports of adolescent behavior problems and child reports of relationship quality. Within-reporter correlations are compared to between-reporter correlations using contrasts for overlapping associations (Meng et al. 1992).

Dyadic analyses describe associations between adolescent behavior problems and mother-child relationship quality, separating variance shared by mothers and children from variance unique to mothers and unique to children. The structural equation model in Figure 1 was estimated with Mplus version 5.1 (Muthén and Muthén, 1998-2007) using a covariance matrix as input. The model includes one dyadic correlation and two individual correlations. The dyadic path describes the linear association between latent variables representing perceptions of adolescent behavior problems shared by mothers and children and perceptions of mother-child relationship quality shared by mothers and children. Individual paths describe correlations between adolescent behavior problems and mother-child relationship quality that are unique to child reports and associations that are unique to maternal reports. To identify the model, the two factor loadings for each latent variable are set to 1 and the error variances of the observed measures are constrained to be equal between reporters. While these equality 
Fig. 1 Dyadic and individual correlations between adolescent behavior problems and mother-child relationship quality: a common-fate model. Note. Paths with same subscripts are constrained to be equal

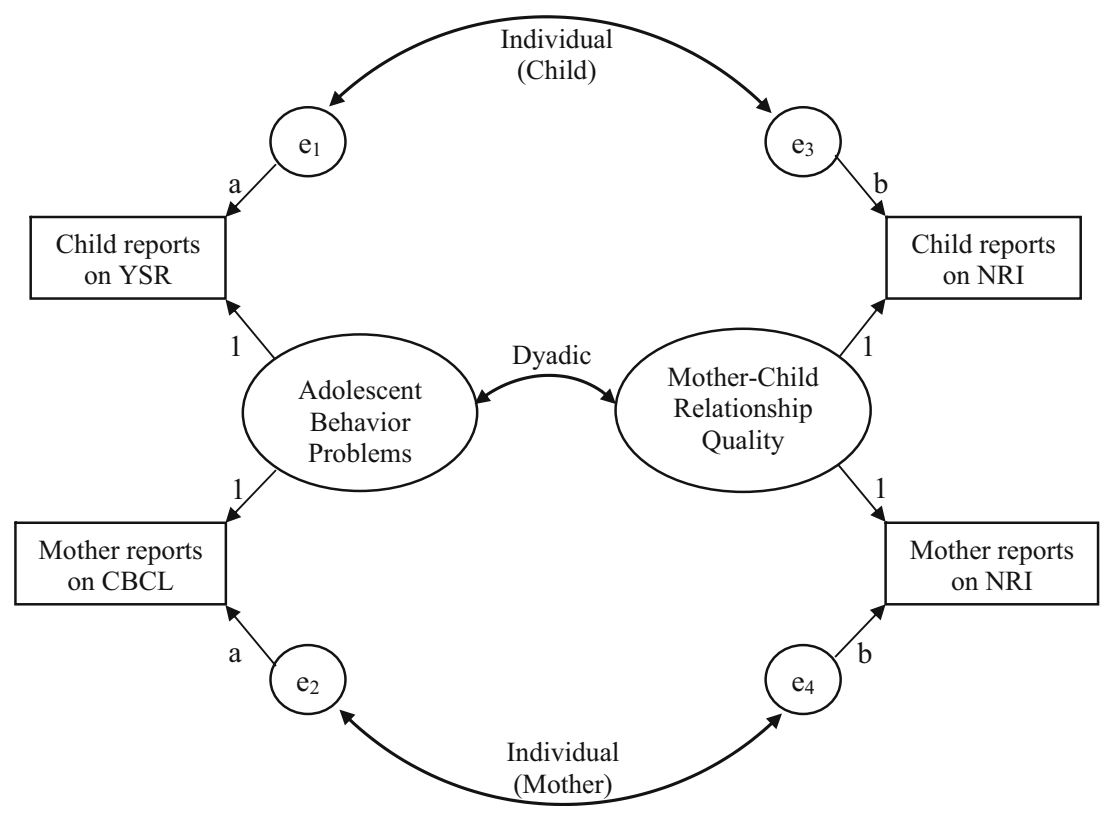

constraints mandate the evaluation of unstandardized correlation coefficients, it should be noted that this specification results in standardized factor loadings for each latent variable that are the square root of the intraclass correlation for each dyadic measure; the corresponding error variances in the standardized solution are the square root of one minus the intraclass correlation (see Gonzalez and Griffin 1999).

A series of additional model constraints were used to test three types of differences in the magnitude of the dyadic and individual correlations: between the dyadic and individual mother correlation, between the dyadic and individual child correlation, and between the individual mother and individual child correlations. $\chi^{2}$ tests identified differences between models with and without these constraints. Supplemental contrasts failed to reveal statistically significant sex differences at levels greater than chance in any set of correlations.

\section{Results}

\section{Preliminary Analyses}

As indicated in Table 1, adolescents reported more behavior problems and relationship negativity than mothers. Paired $t$ tests revealed statistically significant differences between mother and child reports on all variables except social problems.

Intraclass correlations represent the total variance accounted for by an association. These intraclass correlations indicated that approximately $50 \%$ of the variance in individual reports of relationship quality and $25 \%$ of the variance in reports of adolescent behavior problems was shared between mothers and children. Although neither thought problems nor delinquent behaviors reached conventional levels of statistical significance, a liberal test of interdependence $(p<0.25)$ is recommended to identify variables that qualify for inclusion in common fate analyses (Kenny and La Voie 1985). As a consequence, only thought problems were omitted from subsequent analyses.

Simple Correlations Between Adolescent Behavior Problems and Mother-Child Relationship Quality

Table 2 describes simple correlations between adolescent behavior problems and mother-child relationship quality, separately within maternal reports and within child reports, and also between child reports of adolescent behavior problems and maternal reports of relationship quality (childmother correlations) and between maternal reports of adolescent behavior problems and child reports of relationship quality (mother-child correlations). Within reporters, all adolescent behavior problems variables were positively correlated with relationship negativity (average $r=0.38$ for maternal reports and 0.25 for adolescent reports) and inversely correlated with relationship support (average $r=$ -0.44 for maternal reports and -0.34 for adolescent reports). Associations within maternal reports were somewhat higher than those within child reports, but correlation contrasts failed to detect any differences, with the exception of the association between relationship negativity and adolescent withdrawn behaviors $(z=2.78, p=0.005)$.

Between-reporter correlations also indicated that adolescent behavior problems were positively associated with relationship negativity (average $r=0.27$ for child-mother 
Table 1 Means, Standard

Deviations, and Intraclass Correlations for Child and Mother Reports of Adolescent Behavior Problems (YSR and CBCL) and Mother-Child Relationship Quality (NRI)

$N=72$ dyads. $d=$ effect size of mean-level differences between mother and child reports. $\mathrm{ICC}=$ Intraclass correlations between maternal reports and child reports. Relationship negativity and support scales ranged from 1 (little or none) to 5 (the most) Adolescent behavior problem scales (mother reports of CBCL and child reports of YSR) ranged from 0 (never) to 2 (often). ${ }^{+} p<0.25$. ${ }^{*} p<0.05$. ** $p<0.01$

\begin{tabular}{|c|c|c|c|c|c|c|}
\hline \multirow[t]{2}{*}{ Measure } & \multicolumn{2}{|c|}{ Child report } & \multicolumn{2}{|c|}{ Mother report } & \multirow[t]{2}{*}{$d$} & \multirow[t]{2}{*}{$I C C$} \\
\hline & $M$ & $(S D)$ & $M$ & $(S D)$ & & \\
\hline \multicolumn{7}{|l|}{ Mother-child relationship } \\
\hline Relationship negativity & 2.65 & $(1.16)$ & 2.35 & $(1.00)$ & $0.28^{*}$ & $0.51^{* *}$ \\
\hline Relationship support & 4.17 & $(0.71)$ & 4.09 & $(0.56)$ & 0.12 & $0.50^{* *}$ \\
\hline \multicolumn{7}{|c|}{ Adolescent behavior problems } \\
\hline Externalizing symptoms & 0.33 & $(0.23)$ & 0.21 & $(0.22)$ & $0.53^{* *}$ & $0.24^{*}$ \\
\hline Aggressive behaviors & 0.37 & $(0.27)$ & 0.27 & $(0.93)$ & $0.35^{*}$ & $0.28^{* *}$ \\
\hline Delinquent behaviors & 0.24 & $(0.23)$ & 0.12 & $(0.75)$ & $0.64^{* *}$ & $0.15^{+}$ \\
\hline Internalizing symptoms & 0.31 & $(0.24)$ & 0.16 & $(0.15)$ & $0.75^{* *}$ & $0.26^{*}$ \\
\hline Anxiety/depression & 0.27 & $(0.26)$ & 0.18 & $(0.78)$ & $0.39^{* *}$ & $0.26^{*}$ \\
\hline Somatic complaints & 0.29 & $(0.27)$ & 0.13 & $(0.51)$ & $0.71^{* *}$ & $0.27^{*}$ \\
\hline Withdrawn behaviors & 0.38 & $(0.32)$ & 0.19 & $(0.86)$ & $0.70^{* *}$ & $0.27^{*}$ \\
\hline \multicolumn{7}{|l|}{ Other symptoms } \\
\hline Attention problems & 0.40 & $(0.33)$ & 0.23 & $(0.81)$ & $0.57^{* *}$ & $0.35^{* *}$ \\
\hline Social problems & 0.31 & $(0.25)$ & 0.25 & $(0.18)$ & 0.23 & $0.34^{* *}$ \\
\hline Thought problems & 0.30 & $(0.37)$ & 0.09 & $(0.21)$ & $0.73^{* *}$ & 0.01 \\
\hline Total symptoms & 0.47 & $(0.10)$ & 0.36 & $(0.08)$ & $1.21^{* *}$ & $0.29^{* *}$ \\
\hline
\end{tabular}

correlations and 0.28 for mother-child correlations) and inversely associated with relationship support (average $r=$ -0.26 for child-mother correlations and -0.25 for motherchild). Within-reporter correlations significantly differed in magnitude from between reporter correlations in 30 out of 80 contrasts. In each case, within reporter correlations were greater than between reporter correlations ( $z$-scores for significant contrasts ranged from 1.97 to 4.62 ). Of these differences, 23 involved relationship support and 7 involved relationship negativity; 22 involved withinreporter correlations from mother reports and 8 involved within-reporter correlations from child reports. Statistically significant differences are represented in Table 2 by differing subscripts. For each behavior problem, within-

Table 2 Simple Correlations Between Adolescent Behavior Problems and Mother-Child Relationship Quality

\begin{tabular}{|c|c|c|c|c|c|c|c|c|}
\hline \multirow[t]{3}{*}{ Adolescent behavior problems } & \multicolumn{4}{|c|}{ Relationship negativity } & \multicolumn{4}{|c|}{ Relationship support } \\
\hline & \multicolumn{2}{|c|}{ Within reporter } & \multicolumn{2}{|c|}{ Between reporters } & \multicolumn{2}{|c|}{ Within reporter } & \multicolumn{2}{|c|}{ Between reporters } \\
\hline & Child & Mother & $\begin{array}{l}\text { Child-- } \\
\text { Mother }\end{array}$ & $\begin{array}{l}\text { Mother- } \\
\text { Child }\end{array}$ & Child & Mother & $\begin{array}{l}\text { Child-- } \\
\text { Mother }\end{array}$ & $\begin{array}{l}\text { Mother- } \\
\text { Child }\end{array}$ \\
\hline Total symptoms & $0.31^{* *}$ & $0.48_{\mathrm{a}}^{* *}$ & $0.32_{\mathrm{b}}{ }^{* *}$ & $0.39^{* *}$ & $-0.42_{\mathrm{a}}^{* *}$ & $-0.55_{\mathrm{a}}^{* *}$ & $-0.30_{\mathrm{b}}{ }^{* *}$ & $-0.29{ }^{*}$ \\
\hline Externalizing symptoms & $0.39^{* *}$ & $0.48^{* * *}$ & $0.30_{\mathrm{b}}{ }^{* *}$ & $0.39^{* *}$ & $-0.49^{* *}$ & $-0.46_{\mathrm{a}}^{* *}$ & $-0.32_{\mathrm{b}}{ }^{* *}$ & $-0.22_{\mathrm{b}}$ \\
\hline Aggressive behaviors & $0.38^{* *}$ & $0.48_{\mathrm{a}}^{* *}$ & $0.29_{\mathrm{b}}^{*}$ & $0.42^{* *}$ & $-0.40_{\mathrm{a}}^{* *}$ & $-0.46_{\mathrm{a}}^{* *}$ & $-0.28_{\mathrm{b}}^{*}$ & $-0.24_{\mathrm{b}}{ }^{*}$ \\
\hline Delinquent behaviors & $0.39^{* *}$ & $0.37^{* *}$ & $0.38^{* *}$ & $0.26^{*}$ & $-0.48^{* *}$ & $-0.41_{\mathrm{a}}^{* *}$ & $-0.23_{\mathrm{b}}^{*}$ & $-0.16_{\mathrm{b}}$ \\
\hline Internalizing symptoms & 0.15 & $0.36^{* *}$ & $0.26^{*}$ & $0.25^{*}$ & $-0.32^{* *}$ & $-0.42^{* *}$ & $-0.29^{*}$ & $-0.32^{* *}$ \\
\hline Anxiety/depression & 0.17 & $0.31^{* *}$ & $0.27^{*}$ & 0.21 & $-0.25^{*}$ & $-0.39^{* *}$ & $-0.24_{\mathrm{b}}^{*}$ & $-0.28^{*}$ \\
\hline Somatic complaints & 0.14 & 0.11 & 0.19 & 0.04 & -0.15 & $-0.27^{*}$ & -0.17 & $-0.26^{*}$ \\
\hline Withdrawn behaviors & 0.13 & $0.41_{\mathrm{a}}^{* *}$ & $0.16_{\mathrm{b}}$ & $0.25_{\mathrm{b}}^{*}$ & $-0.35^{* *}$ & $-0.48_{\mathrm{a}}^{* *}$ & $-0.29 \mathrm{~b}^{*}$ & $-0.24_{\mathrm{b}}^{*}$ \\
\hline \multicolumn{9}{|l|}{ Other symptoms } \\
\hline Attention problems & $0.35^{* *}$ & $0.40_{\mathrm{a}}^{* *}$ & $0.27_{\mathrm{b}}^{*}$ & $0.34^{* *}$ & $-0.36_{\mathrm{ab}}{ }^{* *}$ & $-0.47_{\mathrm{a}}^{* *}$ & $-0.28_{\mathrm{bc}}{ }^{*}$ & $-0.22_{\mathrm{c}}$ \\
\hline Social problems & 0.17 & $0.38_{\mathrm{a}}^{* *}$ & $0.25_{\mathrm{b}}^{*}$ & $0.28^{*}$ & -0.16 & $-0.46_{\mathrm{a}}^{* *}$ & $-0.16_{\mathrm{b}}$ & $-0.23_{\mathrm{b}}{ }^{*}$ \\
\hline
\end{tabular}

$N=72$ dyads. Child-Mother correlations include child reports of adolescent behavior problems (YSR) and mother reports of relationship quality (NRI). Mother-Child correlations include mother reports of adolescent behavior problems (CBCL) and child reports of relationship quality (NRI). Within rows, different subscripts denote within-reporter correlations that significantly differ $(p<0.05)$ from corresponding between-reporter correlations in contrasts that were conducted separately for relationship negativity and relationship support. ${ }^{*} p<0.05$. ${ }^{* *} p<0.01$ 
reporter correlations were contrasted with between-reporter correlations, separately for relationship negativity and relationship support. Consider total problems by way of example. The within-reporter (mother) correlation between total behavior problems and relationship negativity was greater than the between-reporter correlation (childmother). Both within-reporter correlations between total behavior problems and relationship support were greater than both between-reporter correlations.

Dyadic and Individual Correlations Between Adolescent Behavior Problems and Mother-Child Relationship Quality

Table 3 describes associations between adolescent behavior problems and mother-child relationship quality that are shared across mother and child reports (dyadic correlations) and that are unique to mother reports and child reports (individual correlations). The $\chi^{2}$ statistic (with 3 degrees of freedom) was nonsignificant for each model (range $=2.23$ to $7.51, p=0.53$ to 0.06 ), indicating the dyadic models adequately fit the observed data. The average CFI (Comparative Fit Index) was 0.97 (range 0.89 to 1.00 ), and the average RMSEA (Root Mean Square Error of Approximation) was 0.06 (range 0.00 to 0.11 ). CFI values above 0.90 and RMSEA values of less than 0.08 are generally considered as indicators of adequate model fit; CFI values above 0.95 and RMSEA values less than 0.05 represent good fit (Hu and Bentler 1999; Browne and Cudeck 1993). The Appendix presents the model fit statistics for each of the 20 common fate models. The standardized factor loadings of the latent variables, which correspond to the square root of the intraclass correlations presented in Table 1, ranged from 0.39 (for delinquency) to 0.71 (for relationship support).
Except for the association between somatic complaints and negativity, dyadic correlations revealed statistically significant associations between adolescent behavior problems and relationship negativity (average $r=0.27$ ), and between adolescent behavior problems and relationship support (average $r=-0.24$ ). Behavior problems were not associated with negativity at the individual level (average $r=0.12$ for mothers and average $r=-0.05$ for children), with the exception of the individual mother correlation between negativity and withdrawn behaviors. Individual correlations revealed inverse associations between behavior problems and relationship support; 7 out of 10 mother correlations and 2 out of 10 child correlations reached statistically significance (average $r=-0.21$ for mothers and average $r=-0.07$ for children). Similar to the comparisons of the simple correlations, differences between dyadic and individual correlations are represented in Table 3 by different subscripts for associations involving relationship negativity and for associations involving relationship support.

Dyadic correlations between negativity and behavior problems were always greater in magnitude than individual correlations; these differences reached statistical significance in 7 out of 10 comparisons involving child correlations (average $\Delta \chi^{2}(1)=6.26, p=0.012$ ), but they were nonsignificant in all comparisons involving mother correlations. There were no statistically significant differences between dyadic correlations and individual correlations in associations between relationship support and adolescent behavior problems. Individual mother correlations were generally larger in magnitude than individual child correlations, but there were only two instances where these differences reached statistical significance: relationship negativity and withdrawn behaviors, $\Delta \chi^{2}(1)=7.96, p=0.005$; and relationship support and social problems, $\Delta \chi^{2}(1)=4.92, p=0.027$.
Table 3 Dyadic and Individual Correlations Between Adolescent Behavior Problems and Mother-Child Relationship Quality

$N=72$ dyads. Within rows, different subscripts denote dyadic correlations that significantly differ $(p<0.05)$ from corresponding individual correlations in contrasts that were conducted separately for relationship negativity and relationship support. ${ }^{*} p<0.05$. ${ }^{* *} p<0.01$.

\begin{tabular}{|c|c|c|c|c|c|c|}
\hline \multirow[t]{3}{*}{ Adolescent behavior problems } & \multicolumn{3}{|c|}{ Relationship negativity } & \multicolumn{3}{|c|}{ Relationship support } \\
\hline & \multirow[t]{2}{*}{ Dyadic } & \multicolumn{2}{|c|}{ Individual } & \multirow[t]{2}{*}{ Dyadic } & \multicolumn{2}{|c|}{ Individual } \\
\hline & & Child & Mother & & Child & Mother \\
\hline Total symptoms & $0.34^{* *}$ & $-0.05_{\mathrm{b}}$ & 0.11 & $-0.28^{* *}$ & -0.10 & $-0.25^{* *}$ \\
\hline Externalizing symptoms & $0.34_{\mathrm{a}}^{* *}$ & $0.03_{\mathrm{b}}$ & 0.15 & $-0.26^{* *}$ & $-0.18^{*}$ & $-0.24^{*}$ \\
\hline Aggressive behaviors & $0.35_{\mathrm{a}}^{* *}$ & $0.02_{\mathrm{b}}$ & 0.10 & $-0.25^{* *}$ & -0.12 & $-0.21^{*}$ \\
\hline Delinquent behaviors & $0.31_{\mathrm{a}}^{* *}$ & $0.02_{\mathrm{b}}$ & 0.12 & $-0.18^{*}$ & $-0.23^{*}$ & $-0.28^{* *}$ \\
\hline Internalizing symptoms & $0.25_{\mathrm{a}}^{*}$ & $-0.14_{b}$ & 0.10 & $-0.29^{* *}$ & 0.01 & -0.16 \\
\hline Anxiety/depression & $0.24^{*}$ & -0.14 & 0.11 & $-0.26^{* *}$ & 0.01 & -0.17 \\
\hline Somatic complaints & 0.11 & 0.01 & 0.03 & $-0.21^{*}$ & 0.06 & -0.08 \\
\hline Withdrawn behaviors & $0.22^{*}$ & -0.19 & $0.20^{*}$ & $-0.26^{* *}$ & -0.05 & $-0.24^{* *}$ \\
\hline \multicolumn{7}{|l|}{ Other symptoms } \\
\hline Attention problems & $0.30_{\mathrm{a}}^{* *}$ & $0.03_{\mathrm{b}}$ & 0.12 & $-0.24^{*}$ & -0.09 & $-0.25^{* *}$ \\
\hline Social problems & $0.27_{\mathrm{a}}^{* *}$ & $-0.10_{\mathrm{b}}$ & 0.14 & $-0.21^{*}$ & 0.04 & $-0.25^{* *}$ \\
\hline
\end{tabular}


Figure 2a presents the model examining total problems of adolescents and mother-child relationship negativity. Mothers and children shared views that adolescent problem behaviors are associated with relationship negativity. The dyadic correlation was significantly higher in magnitude than the individual child correlation; the individual mother correlation was non-significant, but did not differ from either the dyadic and individual child correlations. The pattern of association indicates that maternal, and particularly child reports, are not inflated by shared reporter bias. Figure $2 \mathrm{~b}$ presents the model estimating associations between total behavior problems and mother-child relationship support. Mothers and children shared views that adolescent problems are inversely associated with relationship support. However, mothers' perceptions of this link uniquely predict additional variance, suggesting the withinpartner correlation for maternal reports is substantially inflated due to shared reporter variance; the nonsignificant individual child correlation suggests this bias is less pronounced within adolescent reports.

\section{Discussion}

In this study, mother and adolescent reports of child behavior problems and mother-child relationship qualities were not independent. Consistent with other studies of normative populations, adolescents in the present study described more adjustment difficulties and presented a less rosy view of their relationship than did mothers (Noller and Callan 1988). These mean-level differences should not be overstated: Intraclass correlations indicated that similarity between mother and child perceptions accounts for approximately $50 \%$ of the variance in relationship quality and $25 \%$ of the variance in adolescent behavior problems. Simple correlations between indices of adolescent behavior problems and mother-child relationship quality tended to be more robust within mother reports than between mother and child reports. We sought to determine if this was a product of same-reporter variance bias, so within-reporter correlations were decomposed into associations that described views shared by participants (dyadic correlations) and associations that described views unique to mothers and children (individual correlations).

The findings suggest that conventional within-reporter correlations contain variance that is not necessarily biased but may instead more accurately be ascribed to views that are shared by both reporters. Adjustments for shared views resulted in the elimination of almost all statistically significant correlations between relationship negativity and adolescent behavior problems. This pattern of results suggests that neither mother nor child views of the associations between adolescent behavior problems and relationship negativity are inflated by shared reporter variance. The inclusion of shared views also eliminated all but two of the associations between child views of support and child views of adolescent behavior problems, but it did little to alter the pattern of associations between maternal views of support and maternal views of adolescent behavior problems. Unlike associations involving relationship negativity, the pattern of results for links between behavior problems and relationship support indicate that maternal reports are more susceptible to biases related to same reporter variance than child reports.

We have long known that studies that rely on a single rater for assessments of predictor and outcome variables yield stronger effects than studies that rely on different raters for each. A typical study of maternal and adolescent views reveals correlations from externalizing behaviors to support and conflict in the 0.40 to 0.50 range within reporters and correlations in the 0.10 to 0.20 range between reporters (Barrera et al. 1993). We replicated this pattern of results and then went a step further, estimating the degree to which within-reporter correlations are inflated by same reporter variance. To this end, we conducted common fate dyadic analyses that decomposed associations into variance shared across reporters and variance unique to each reporter. Dyadic correlations accounted for most of the variance in these associations; individual correlations revealed that biases involving common reporter variance to be an issue only in associations involving maternal reports of relationship support and adolescent behavior problems. Simple correlations offered no hint of that these associations might differ. These findings underscore the conclusion that same reporter variance bias should not be assumed in the absence of dyadic analyses that simultaneously assess shared views and unilateral views.

We are not the first to report that adolescent behavior problems are inversely associated with mother-child support and positively associated with mother-child negativity (see Laursen and Collins 2009, for review). The common fate approach eliminates the bewildering array of competing correlations within and across reporters, providing a parsimonious interpretation of the data. When considered apart, associations involving both parent and child reports can be difficult to interpret, but when considered jointly they tend to form a more coherent picture. Put simply, mothers and children share the view that most indices of behavior problems are linked to relationships negativity and support. Unique views yielded somewhat different patterns of statistical significance, but correlation contrasts between individual mother and child associations indicated that the magnitude of these differences rarely reached statistical significance.

These findings do not mean that parent and child views are interchangeable, only that they tend to yield similar patterns of association. It is noteworthy that differences in 
Fig. 2 a Dyadic and individual correlations between adolescent total behavior problem symptoms and mother-child relationship negativity. Note. ${ }^{*} p<0.01$. b dyadic and individual correlations between adolescent total behavior problem symptoms and mother-child relationship support. Note. ${ }^{*} p<0.01$
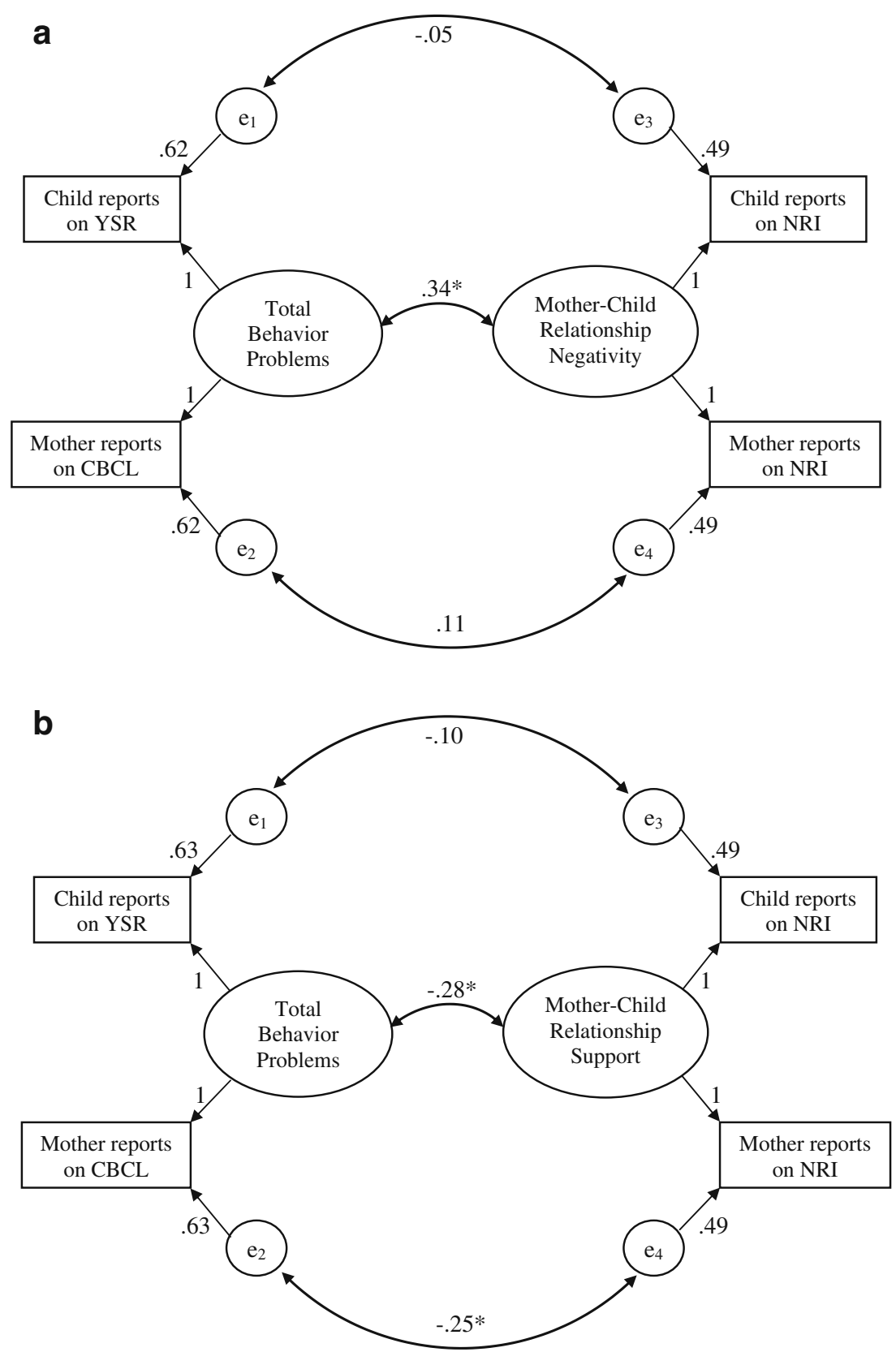

individual correlations arose in the realm of internalizing syndromes and its subscales, with findings suggesting that mothers uniquely associate these behaviors with more relationship negativity and less support than do children. This is perhaps not surprising given that mothers are less privy to the internal states of their children than to their external states. These findings are in line with previous studies indicating that the congruence of mother and child views varies as a function of the salience of the problem behavior, the degree to which problems can be observed by both partners, and the willingness of each partner to report problems (Herjanic and Reich 1982; Karver 2006). The present study adds to this literature by providing prelimi- nary evidence that shared and unique views of adolescent behavior problems are differentially related to mother-child relationship characteristics.

Several limitations must be acknowledged. First, low maternal participation rates significantly reduced the sample, precluding the use of more complex multivariate analyses and the examination of a host of important moderator analyses. Although adolescents whose mothers participated in the study differed little from those whose mothers did not participate, generalizability remains an appropriate concern. Second, adolescent and mother reports were not collected on the same day. We found no evidence that the timing of data collection influenced the results, but 
there can be little doubt that failure to gather simultaneous reports lowered estimates of shared perceptions and increased the likelihood that unique perceptions were responsible for the variance in reports. Third, maternal psychopathology was not assessed in this study. Previous findings suggest that similarity between mother and child reports differs as a function of mother's behavior problems, particularly depressive symptoms (Briggs-Gowan et al. 1996; Treutler and Epkins 2003). Fourth, father-child relationships were not considered in our study. Recent evidence suggests links between adolescents' depressive symptoms and relationship characteristics are similar in mother-child and father-child relationships (Sheeber et al. 2007), but it would be imprudent to speculate as to whether dyadic analyses would yield the same pattern of results. Finally, our analyses failed to consider nonlinear associations between behavior problems and relationship characteristics. Links between perceptions of relationship quality and behavior problems may be especially pronounced among troubled youth with poor relationships (Laursen and Hafen 2010).
Absent from our list of limitations is the fact that our study was based entirely on self-reports. Self-reports have plenty of drawbacks, of course, and they ought not be considered a substitute for observations. Nevertheless, we have demonstrated that some of the potential biases of self-report data may be ameliorated with the appropriate analytic tools. Participant perceptions are at the core of family relationships (Noller and Callan 1988) and we must embrace both insider and outsider views of interactions in order to understand how relationships shape participants (Olson 1977). We have described an important analytic tool that takes reports from both partners in a relationship and disentangles shared views from those that are unique to individuals. As such, it has the potential to offer new insight into the role of shared and unique perceptions in family relationships and family interactions.

Open Access This article is distributed under the terms of the Creative Commons Attribution Noncommercial License which permits any noncommercial use, distribution, and reproduction in any medium, provided the original author(s) and source are credited.

\section{Appendix}

Table 4 Model Fit Statistics for the Common Fate Models for Adolescent Behavior Problems and Mother-Child Relationship Quality

\begin{tabular}{|c|c|c|c|c|c|c|c|c|}
\hline \multirow[t]{2}{*}{ Adolescent behavior problems } & \multicolumn{4}{|c|}{ Relationship negativity } & \multicolumn{4}{|c|}{ Relationship support } \\
\hline & $x^{2}$ & $p$-value & CFI & $\overline{\text { RMSEA }}$ & $x^{2}$ & $p$-value & $\mathrm{CFI}$ & $\overline{\text { RMSEA }}$ \\
\hline Total symptoms & 2.58 & 0.461 & 1.00 & 0.000 & 4.25 & 0.236 & 0.978 & 0.076 \\
\hline Externalizing Symptoms & 2.91 & 0.407 & 1.00 & 0.000 & 3.96 & 0.266 & 0.982 & 0.067 \\
\hline Aggressive Behaviors & 4.03 & 0.259 & 0.980 & 0.069 & 5.39 & 0.145 & 0.951 & 0.085 \\
\hline Delinquent behaviors & 4.23 & 0.238 & 0.971 & 0.075 & 4.55 & 0.208 & 0.969 & 0.085 \\
\hline Internalizing symptoms & 2.23 & 0.525 & 1.00 & 0.000 & 3.65 & 0.301 & 0.985 & 0.055 \\
\hline Anxiety/depression & 4.74 & 0.192 & 0.945 & 0.090 & 5.77 & 0.123 & 0.890 & 0.105 \\
\hline Somatic complaints & 6.50 & 0.090 & 0.889 & 0.104 & 7.51 & 0.057 & 0.886 & 0.112 \\
\hline Withdrawn behaviors & 2.51 & 0.473 & 1.00 & 0.000 & 3.61 & 0.307 & 0.974 & 0.053 \\
\hline \multicolumn{9}{|l|}{ Other symptoms } \\
\hline Attention problems & 2.41 & 0.492 & 1.00 & 0.000 & 3.67 & 0.299 & 0.987 & 0.056 \\
\hline Social problems & 3.63 & 0.305 & 0.984 & 0.054 & 5.56 & 0.135 & 0.939 & 0.090 \\
\hline
\end{tabular}

$N=72$ dyads. $\chi^{2}=$ overall chi-square with three degrees of freedom. CFI=Comparative Fit Index. RMSEA=Root Mean Square Error of Approximation. $P$-values less than 0.05 , CFI values greater than 0.90 , and RMSEA values less than 0.08 indicate adequate fit of the estimated model to the observed data.

\section{References}

Achenbach, T. M. (1991). Integrative Guide for the 1991 CBCL/4-18, YSR, \& TRF Profiles. Burlington: University of Vermont.

Achenbach, T. M., McConaughy, S. H., \& Howell, C. T. (1987). Child/adolescent behavioral and emotional problems: implications of cross-informant correlations for situational specificity. Psychological Bulletin, 101, 213-232.
Achenbach, T. M., Connors, C. K., Quay, H. C., Verhulst, F. C., \& Howell, C. T. (1989). Replication of empirically derived syndromes as a basis for taxonomy of child/adolescent psychopathology. Journal of Abnormal Child Psychology, 17, 299-323.

Adams, R. E., \& Laursen, B. (2007). The correlates of conflict: disagreement is not necessarily detrimental. Journal of Family Psychology, 21, 445-458.

Barrera, M., Jr., Chassin, L., \& Rogosch, F. (1993). Effects of social support and conflict on adolescent children of alcoholic and 
nonalcoholic fathers. Journal of Personality and Social Psychology, 64, 602-612.

Briggs-Gowan, M. J., Carter, A. S., \& Schwab-Stone, M. (1996). Discrepancies among mother, child, and teacher reports: examining the contributions of maternal depression and anxiety. Journal of Abnormal Child Psychology, 24, 749-765.

Browne, M. W., \& Cudeck, R. (1993). Alternative ways of assessing fit. In K. A. Bollen \& J. S. Long (Eds.), Testing structural equation models (pp. 136-162). Beverly Hills: Sage.

Caples, H. S., \& Barrera, M. (2006). Conflict, support, and coping as mediators of the relation between degrading parenting and adolescent adjustment. Journal of Youth and Adolescence, 35, 599-611.

Collins, W. A., \& Steinberg, L. (2006). Adolescent development in interpersonal context. In W. Damon \& R. Lerner (Series Ed.) and N. Eisenberg (Vol. Ed.), The handbook of child psychology: Vol 3. Social, emotional and personality development (6th ed. pp. 1003-1067). Wiley, New York.

Cook, W. L., \& Kenny, D. A. (2005). The actor-partner interdependence model: a model of bidirectional effects in developmental studies. International Journal of Behavioral Development, 29, 101-109.

De Los Reyes, A., \& Kazdin, A. E. (2005). Informant discrepancies in the assessment of childhood psychopathology: a critical review, theoretical framework, and recommendations for further study. Psychological Bulletin, 131, 483-509.

Edelbrock, C., \& Costello, A. J. (1988). Convergence between statistically derived behavior problem syndromes and child psychiatric diagnoses. Journal of Abnormal Child Psychology, $16,219-231$

Forehand, R. D., Long, N., \& Hendrick, M. (1987). Family characteristics of adolescents who display overt and covert behavior problems. Journal of Behavior Therapy and Experimental Psychiatry, 18, 325-328.

Furman, W. (1996). The measurement of friendship perceptions: conceptual and methodological issues. In W. M. Bukowski, A. F. Newcomb, \& W. W. Hartup (Eds.), The company they keep: Friendship in childhood and adolescence (pp. 41-65). New York: Cambridge University Press.

Furman, W., \& Buhrmester, D. (1985). Children's perceptions of the personal relationships in their social networks. Developmental Psychology, 21, 1016-1024.

Furman, W., \& Buhrmester, D. (2009). The network of relationships inventory: behavioral systems version. International Journal of Behavioral Development, 33, 470-478.

Gavin, L. A., \& Furman, W. (1996). Adolescent girls' relationships with mothers and best friends. Child Development, 67, 375-386.

Ge, X., Best, K. M., Conger, R. D., \& Simons, R. L. (1996). Parenting behaviors and the occurrence and co-occurrence of adolescent depressive symptoms and conduct problems. Developmental Psychology, 32, 717-731.

Gollob, H. F. (1991). Methods for estimating individual- and grouplevel correlations. Journal of Personality and Social Psychology, 60, 376-381.

Gonzalez, R., \& Griffin, D. (1999). The correlational analysis of dyadlevel data in the distinguishable case. Personal Relationships, 6 , 449-469.

Hafen, C. A., \& Laursen, B. (2009). More problems and less support: early adolescent adjustment forecasts changes in perceived support from parents. Journal of Family Psychology, 22, 193-202.

Herjanic, B., \& Reich, W. (1982). Development of a structured psychiatric interview for children: agreement between child and parent on individual symptoms. Journal of Abnormal Child Psychology, 10, 307-324.

Hollingshead, A. B. (1975). Four factor index of social status. Yale University: Unpublished manuscript.
Hu, L., \& Bentler, P. M. (1999). Cutoff criteria for fit indexes in covariance structure analysis: conventional criteria versus new alternatives. Structural Equation Modeling, 6, 1-55.

Karver, M. S. (2006). Determinants of multiple informant agreement on child and adolescent behavior. Journal of Abnormal Child Psychology, 34, 251-262.

Kenny, D. A. (1995). The effect of nonindependence on significance testing in dyadic research. Personal Relationships, 2, 67-75.

Kenny, D. A., \& La Voie, L. (1985). Separating individual and group effects. Journal of Personality and Social Psychology, 48, 339-348.

Kenny, D. A., Kashy, D. A., \& Cook, W. L. (2006). Dyadic data analysis. New York: Guilford.

Laursen, B. (2005). Dyadic and group perspectives on close relationships. International Journal of Behavioral Development, 29, 97-100.

Laursen, B., \& Collins, W. A. (2009). Parent-child relationships during adolescence. In R. Lerner \& L. Steinberg (Eds.), Handbook of adolescent psychology: Vol. 2. Contextual influences on adolescent development (3rd ed., pp. 3-42). New York: Wiley.

Laursen, B., \& Hafen, C. A. (2010). Future directions in the study of close relationships: Conflict is bad (except when it's not). Social Development.

Laursen, B., Popp, D., Burk, W. J., Kerr, M., \& Stattin, H. (2008). Incorporating interdependence into developmental research: examples from the study of homophily and homogeneity. In N. A. Card, T. D. Little, \& J. P. Selig (Eds.), Modeling dyadic and interdependent data in the developmental and behavioral sciences (pp. 11-37). New York: Routledge.

Meng, X.-L., Rosenthal, R., \& Rubin, D. B. (1992). Comparing correlated correlation coefficients. Psychological Bulletin, 111, 172-175.

Muthén, L. K., \& Muthén, B. O. (1998-2007). Mplus user's guide (5th ed.). Los Angeles: Muthén \& Muthén.

Noller, P., \& Callan, V. J. (1988). Understanding parent-adolescent interactions: perceptions of family members and outsiders. Developmental Psychology, 24, 707-714.

Olson, D. H. (1977). Insiders' and outsiders' views of relationships: research studies. In G. Levinger \& H. Raush (Eds.), Close relationships: Perspectives on the meaning of intimacy. Amherst: University of Massachusetts.

Raghunathan, T. E., Rosenthal, R., \& Rubin, D. B. (1996). Comparing correlated but nonoverlapping correlations. Psychological Methods, $1,178-183$.

Rutter, M., Graham, P., Chadwick, O. F. D., \& Yule, W. (1976). Adolescent turmoil: fact or fiction? Journal of Child Psychology and Psychiatry, 17, 35-56.

Sheeber, L. B., Davis, B., Leve, C., Hops, H., \& Tildesley, E. (2007). Adolescents' relationships with their mothers and fathers: associations with depressive disorder and subdiagnostic symptomatology. Journal of Abnormal Psychology, 116, 144-154.

Silk, J. S., Steinberg, L., \& Morris, A. S. (2003). Adolescents' emotion regulation in daily life: links to depressive symptoms and problem behavior. Child Development, 74, 1869-1880.

Tesser, A., Forehand, R., Brody, G., \& Long, N. (1989). Conflict: the role of calm and angry parent-child discussion in adolescent adjustment. Journal of Social and Clinical Psychology, 8, 317-330.

Tien, J.-Y., Roosa, M. W., \& Michaels, M. (1994). Agreement between parents and child reports on parental behaviors. Journal of Marriage and Family, 56, 341-355.

Tremblay, G., Tremblay, R. E., \& Saucier, J. (2004). The development of parent-child relationship perceptions in boys from childhood to adolescence: a comparison between disruptive and non-disruptive boys. Child and Adolescent Social Work Journal, 21, 407-426.

Treutler, C. M., \& Epkins, C. C. (2003). Are discrepancies among child, mother, and father reports on children's behavior related to parents' psychological symptoms and aspects of parent-child relationships? Journal of Abnormal Child Psychology, 31, 13-27. 